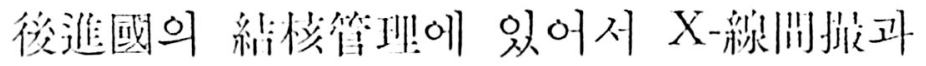

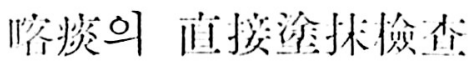

\section{Chest Microfilm Versus Direct Microscopic Sputum Examination in Tuberculosis \\ Control in the Developing Country}

C.T. Hsing, M.D., F.C.C.P.

Taiwan, Republic of China

Discases of the Chest, Vol. 52, No.5, Nov., 1967

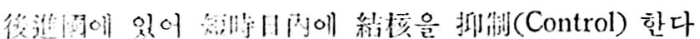
는 것이 사이 이립다는 것은 잘 알려저 있는 거걱인

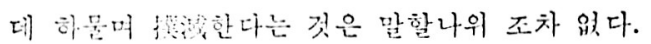

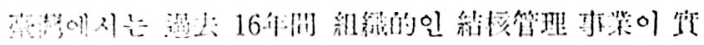

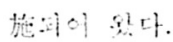

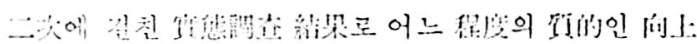

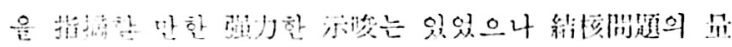

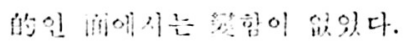

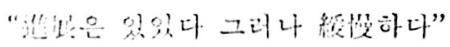

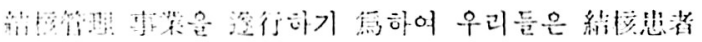

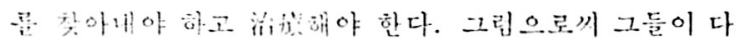

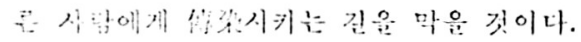

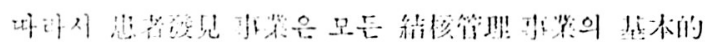

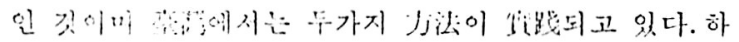

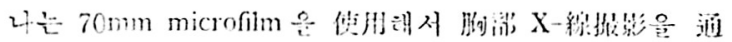

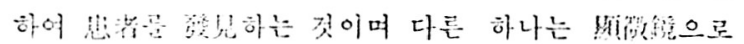

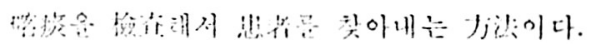

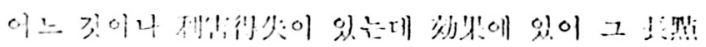

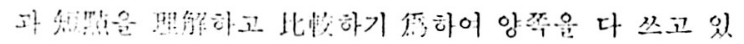

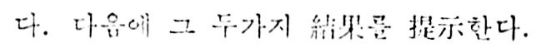

\section{间推层影}

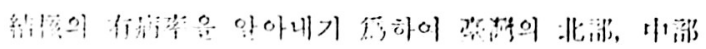

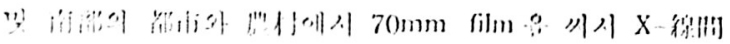

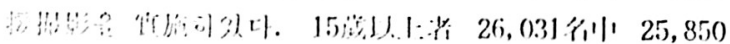

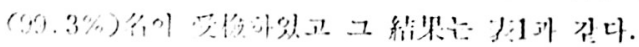

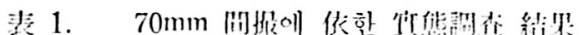

\begin{tabular}{|c|c|}
\hline yot & 26,031 \\
\hline 夌㒀努 & 25,850 \\
\hline 受推果( (\%) & 99.3 \\
\hline 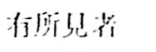 & 1,981 \\
\hline (装i)\% & 7.7 \\
\hline
\end{tabular}

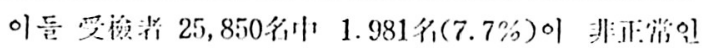

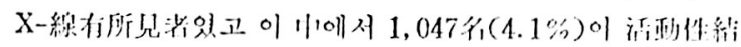
核怒婎(clinically significant tuberculosis)이 며 이둘 1,047

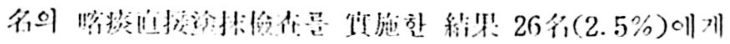

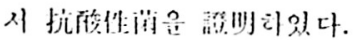

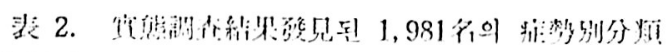

\begin{tabular}{|c|c|c|}
\hline 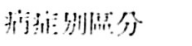 & 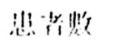 & $\%$ \\
\hline 苦 & 749 & 38.0 \\
\hline 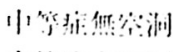 & 182 & 9.1 \\
\hline 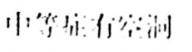 & 19 & 2.4 \\
\hline 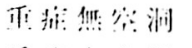 & 26 & 1.3 \\
\hline 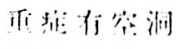 & 39 & 1.9 \\
\hline 泥件䏚脱药 & 2 & 0.01 \\
\hline 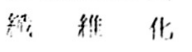 & 816 & 12.0 \\
\hline 非綃 $1 \%$ & 118 & 5.1 \\
\hline
\end{tabular}

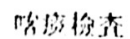

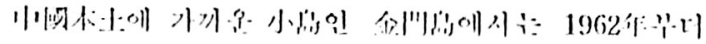

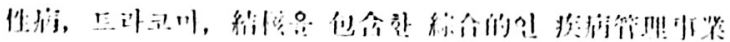




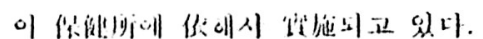

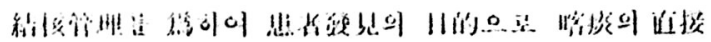

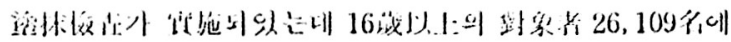

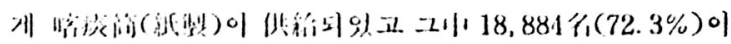

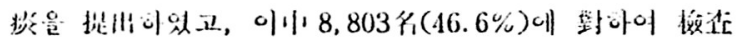
플. 빈이있다. 나미지는 침이었다.

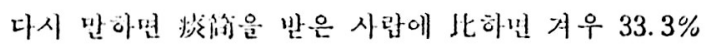

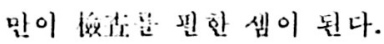

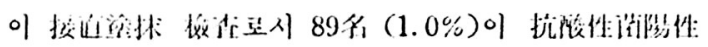
挡있다 (난 3)

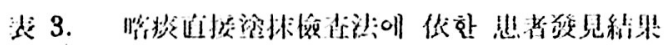

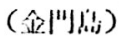

\begin{tabular}{|c|c|}
\hline 16㳚以」:愁人口 & 26,109 \\
\hline 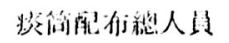 & 26,109 \\
\hline 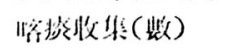 & 18,884 \\
\hline$($ 曻i) & 72.3 \\
\hline 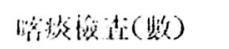 & 8,803 \\
\hline$(\mathrm{fi})$ & 46.6 \\
\hline 临珄渚 & 89 \\
\hline 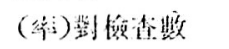 & 1.0 \\
\hline (隹)人口 & 0.3 \\
\hline
\end{tabular}

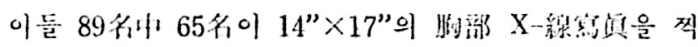

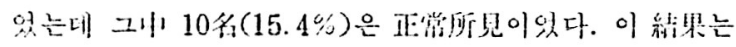

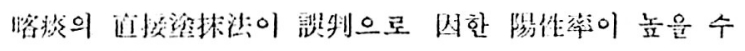
있다는 것을 - 저핫하는 것이다.

\section{討論}

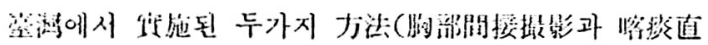

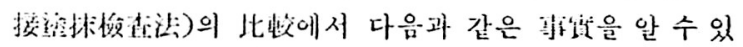
었다.

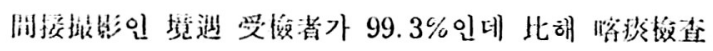
에 있이서는 政察晢의 겨우 $72.3 \%$ 만이 可惨物은 提出 하뎠다.

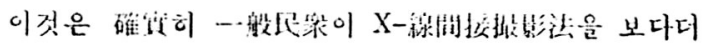
잘 반아든있다는 것이다.

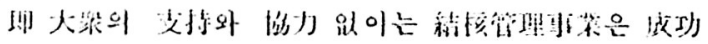

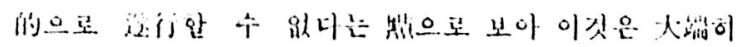
나냈한 것이나.

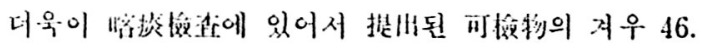

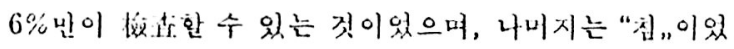

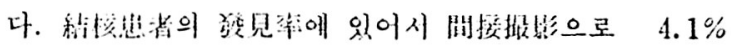

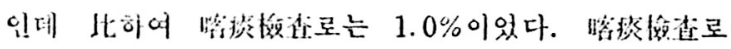

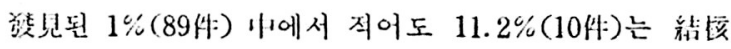

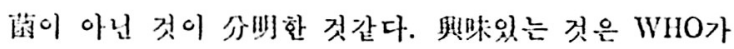

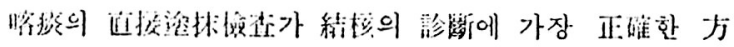

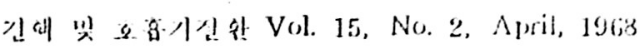

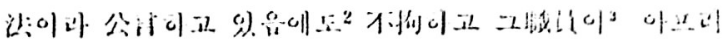

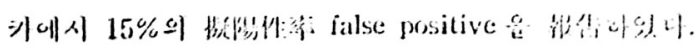

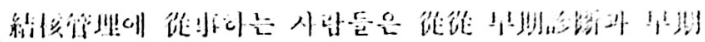

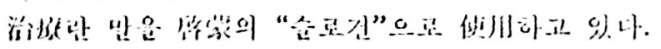

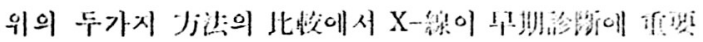

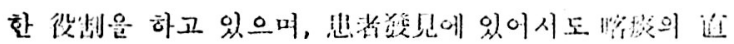

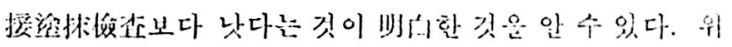

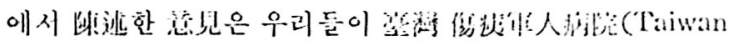

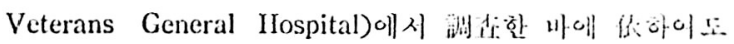

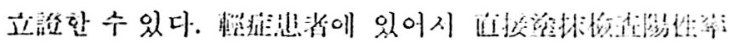

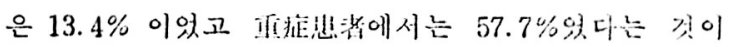

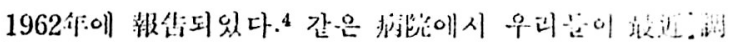

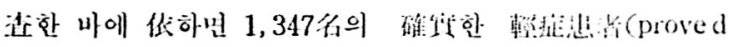
cases of minimal pulmonary tuberculosis) 1 ; 에서 $34 \%$ 이

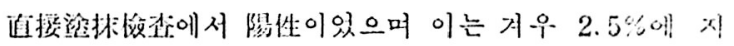
나지 않았다(茨 4).

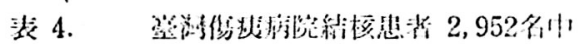

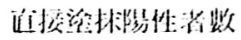

\begin{tabular}{|c|c|c|c|}
\hline 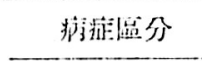 & \multirow{2}{*}{$\frac{\text { 怘消數 }}{1,347}$} & \multicolumn{2}{|c|}{ 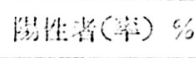 } \\
\hline 倝拉 & & 34 & 2.5 \\
\hline 中1等症严空洞 & 1,073 & 93 & 8.6 \\
\hline 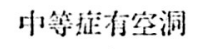 & 242 & 124 & 51.2 \\
\hline 五症 㭃空洞 & 134 & 60 & 44.7 \\
\hline 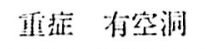 & 156 & 113 & 72.4 \\
\hline
\end{tabular}

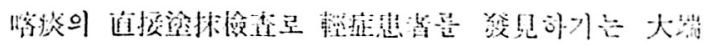

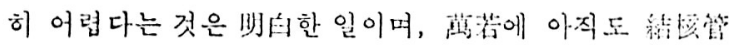

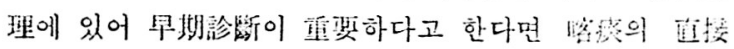

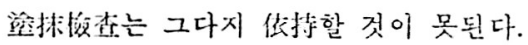

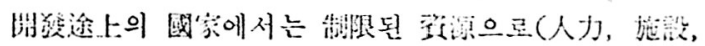

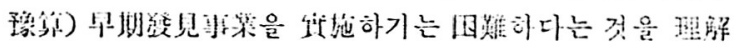
할 수 있다.

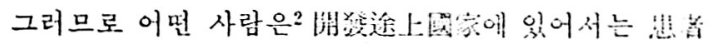

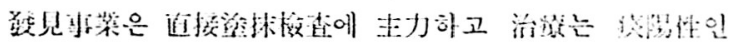

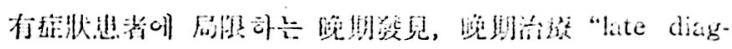

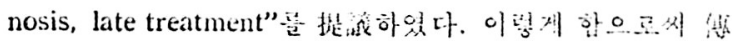

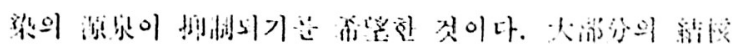
년슨 는

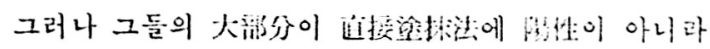

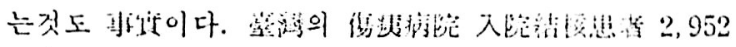

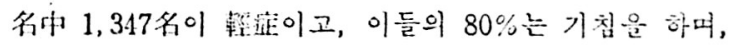

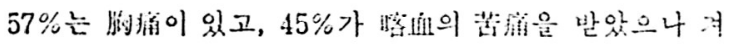

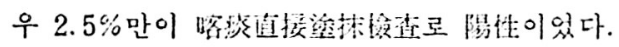

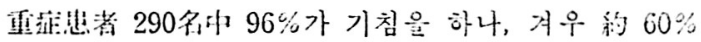

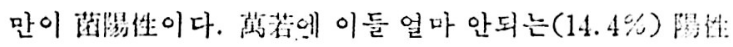




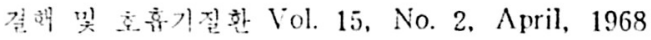

15

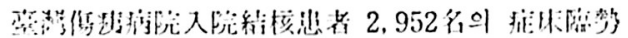

\begin{tabular}{|c|c|c|c|c|c|c|c|c|c|c|c|c|c|c|c|c|c|c|c|}
\hline$n$ & 伿i & 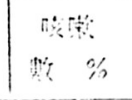 & $\begin{array}{l}M_{0} \\
\text { 慗 }\end{array}$ & & 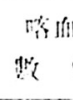 & & 䓡 & $\%$ & 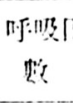 & 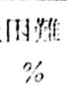 & 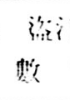 & & 笛: & & 的 & 箈 & 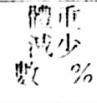 & 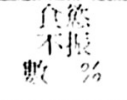 & 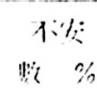 \\
\hline on & & 10788 & 768 & 57 & & 45 & 525 & 39 & & 322 & 471 & 35 & & & 189 & 14 & 283 & $350) 26$ & 242 \\
\hline 中, 算泾 & $1 .:$ & 1,1979 & 77 & 59 & 723 & 55 & 618 & 47 & 28 & 22 & 579 & 4 & 763 & 58 & 263 & 20 & 47336 & 38129 & 171 \\
\hline 花 & 200 & 27896 & 189 & 65 & 143 & 49 & 172 & 59 & 143 & 349 & 123 & 42 & 189 & 65 & 90 & 31 & 13145 & 13346 & 5218 \\
\hline
\end{tabular}

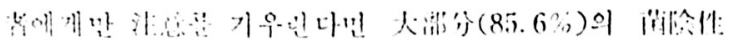

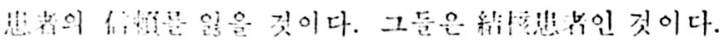
즈리나 그을 이 지 굿하계 뎐 다.

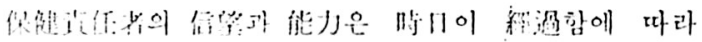

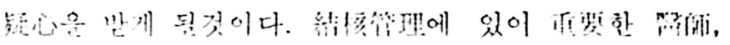

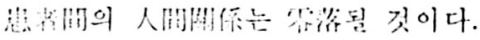

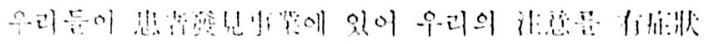

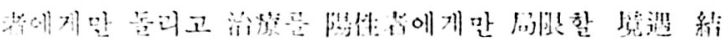

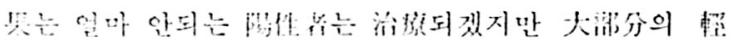

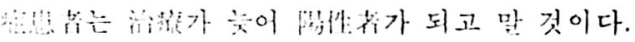

이린

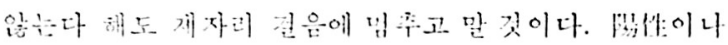

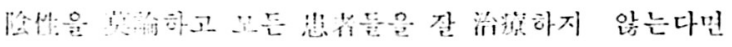

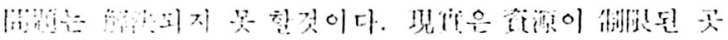

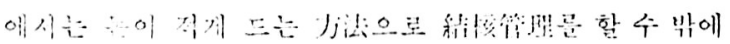

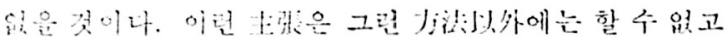

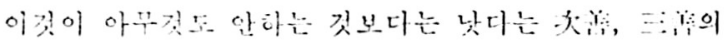

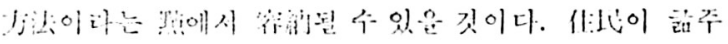

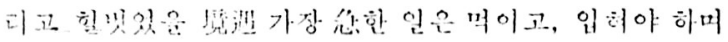

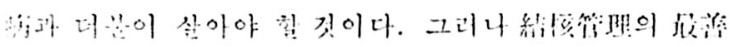

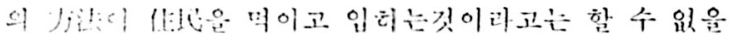
개이나.

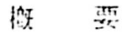

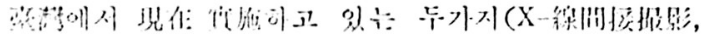

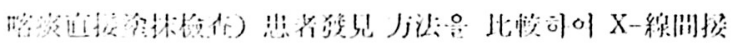

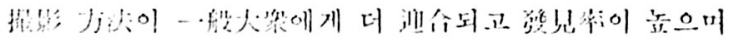

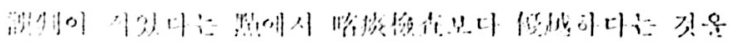
와아아아.

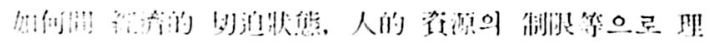

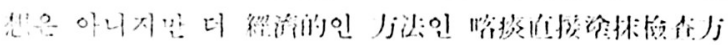

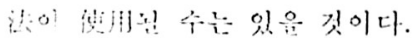

Jy

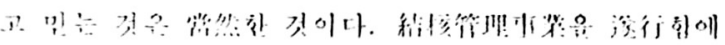

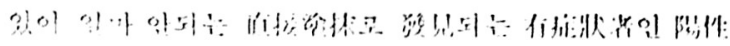

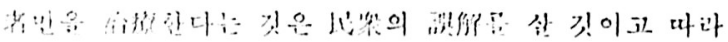

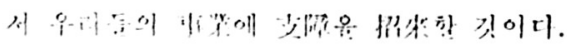

\section{窔考}

Report of the WHO Inter-Regional Scminar on Tuberculosis Control in Deceloping Countrics 에서 case-findrng 에 배한 洲分

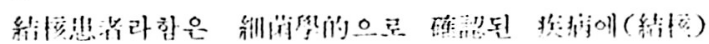

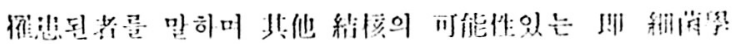

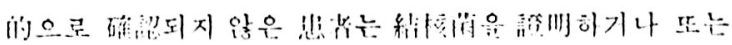

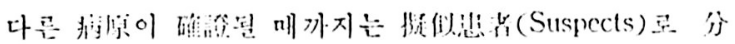
㹦한 다.

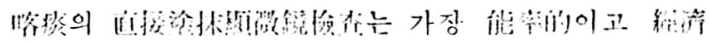

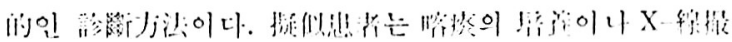
봉갈은 f Centre)로 장/深되이야 한다.

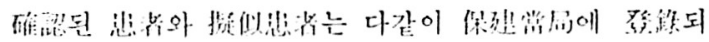

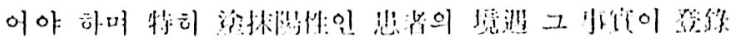
에 빼닌되이야 한다.

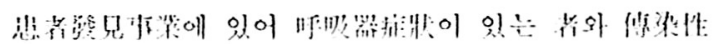

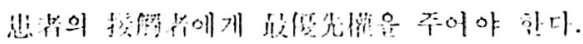

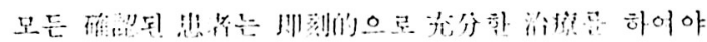

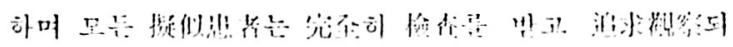

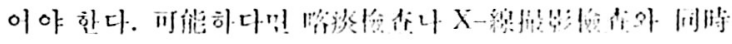

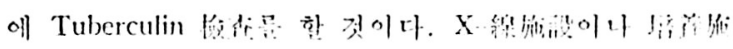

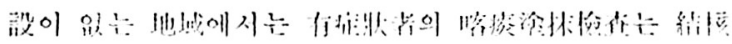

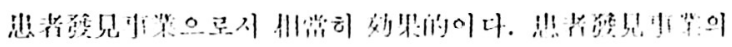

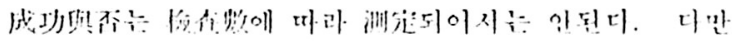

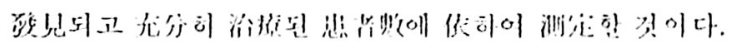

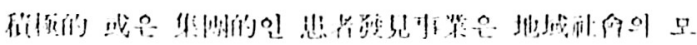

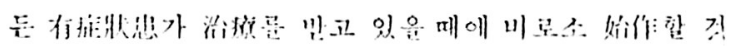

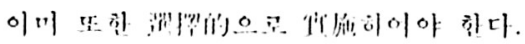

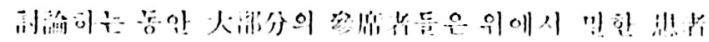

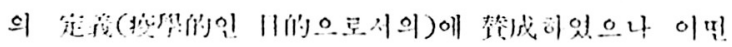

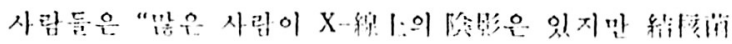

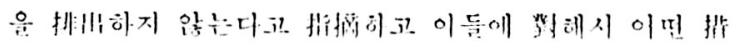

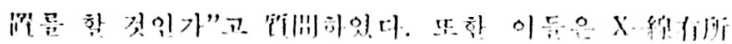

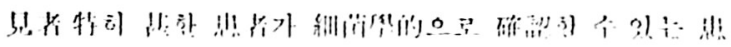

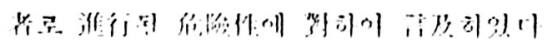

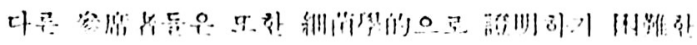

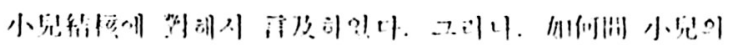




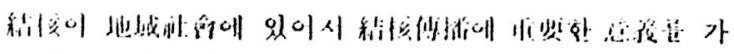

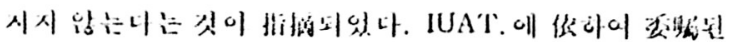

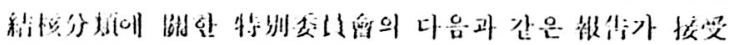

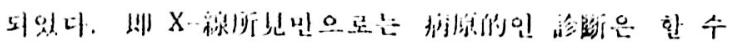

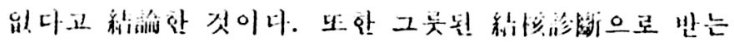

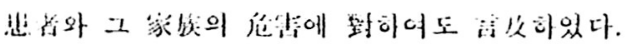

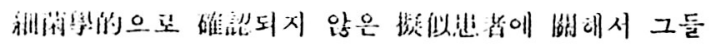

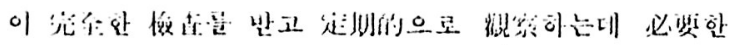

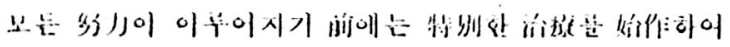

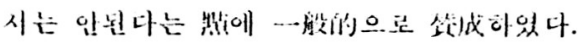

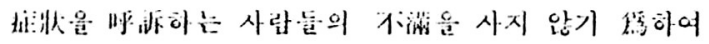

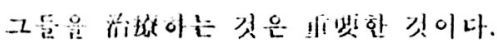

\section{References:}

1. Lin. H.T.: Tuberculosis Problem in Taiuan Results of Second Prevalence Suvey of Taberculosis "SATCA Review 9:2, 1964.

2. Final Report of $(\mathrm{WHO})$ Inter-regional Tuberculosis Seminar, Küala Lampiar, Molaysia November 5-11, 1964

3. ROEISGAARD, E. INVERSEN, E And BI.OGHER G.:

Tuberculosis in Tropical Africa Bull. World, Health Org, 30:459, 1964.

4. ISING. C.T. And MA Y.T.: A Comparative Study of the ejpicacy of the laryngeal suab, bronchial lanage.

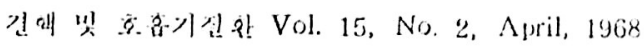
gastric lauage, and direct spatum examination method in detectins tubercle barilli int series of 1,320 Patients Am, Rev, Resp, Dis, 86:16, 1962.

\section{譯 者 註}

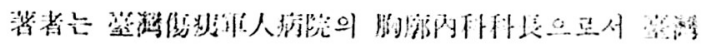

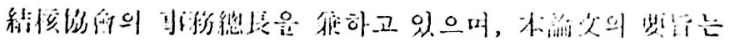

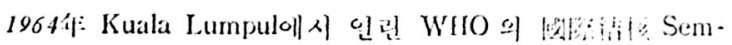

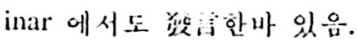

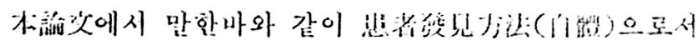

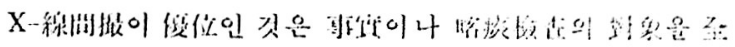

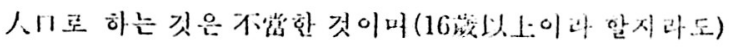

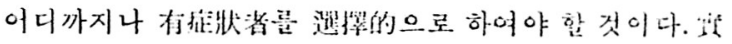

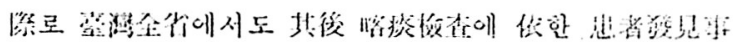

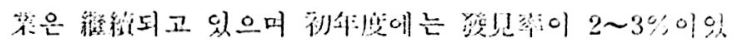

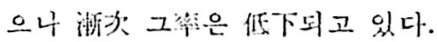

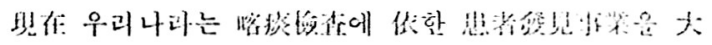

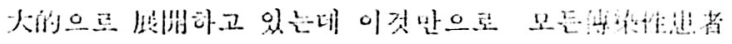

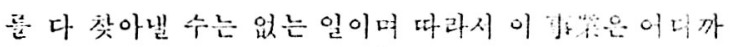

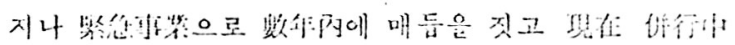

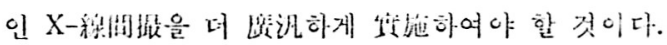

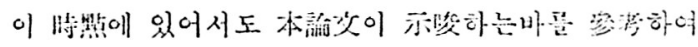

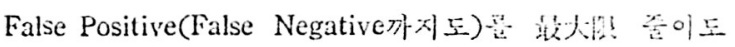

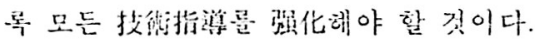

(抄算萑：穼達镜) 